Pflanzliche Extrakte sind ein fester Bestandteil in der Kindermedizin. So ist z.B. der Griff zum Fencheltee vollkommen normal, wenn der Nachwuchs Probleme mit der Verdauung hat. Für gastrointestinale Störungen werden Kamille, Fenchel, Anis, Kümmel, Leinsamen, Flohsamen, Pfefferminze, Karotten oder Heidelbeere eingesetzt. Bei Infektionen der Atemwege kommen Primel, Efeu, Thymian, Königskerze, Spitzwegerich, Huflattich oder Malve zum Einsatz und bei Hauterkrankungen eignen sich Kamille, Eichenrinde, Zauberstrauch, Malve, Ringelblume oder Arnika. Zudem werden psychovegetative Befindlichkeitsstörungen seit Langem mit Hypericum, Passionsblume, Baldrian, Melisse und Hopfen behandelt.

Der Phytotherapie in der Kinderheilkunde stehen allerdings unruhige Zeiten bevor: Demnächst könnte der Fencheltee in die Kritik geraten, weil die meisten Präparate Estragol enthalten. Nach einer Einschätzung der Europäischen Gesundheitsagentur (EMEA) sollte dieser Inhaltsstoff jedoch von besonders empfindlichen Gruppen, wie Kindern und Schwangeren, ferngehalten werden.

Trotz der langen Tradition der Phytotherapie sind nur wenige der Präparate für den Einsatz bei Kindern zugelassen. Die Kinderärzte verschreiben sie zum Gebrauch als «offlabel»: Zwischen 50 und 90\% der Verschreibungen werden so verordnet, schätzt die österreichische Kinderärztin Ulrike Kastner. Auf der «Phytotherapeutika 2012» in Wien berichtete sie über das Spannungsfeld von Phytotherapie und Pädiatrie. Die Kosten für die Behandlung von «off-label use»-Mitteln werden von den Krankenkassen nicht oder nur teilweise übernommen.

Es gibt eine Reihe von Phytotherapeutika, die heute schon Altersbeschränkungen bei der Verwendung in

\title{
Phytotherapie bei Kindern unter Druck
}

Neue Verordnungen sollen für mehr Sicherheit von Kindern sorgen, wenn sie mit neuen pflanzlichen Arzneimitteln behandelt werden. Die Auflagen sind so streng, dass sie bewährte Mittel gefährden und neue Arzneien so gut wie unmöglich machen.

der Kinderheilkunde besitzen. Der Ausschuss für Pflanzliche Arzneimittel der EMEA (HMPC) hat solche Beschränkungen für die Altersstufen 12 und 18 Jahre festgelegt (Görne RC. Z Phytother 2010;31:25-36). Erstaunlicherweise gibt es für keinen Wirkstoff oder Extrakt eine ausreichende Datenlage, die der HMPC ausreichend erscheint, um eine Anwendung bei Kindern unter 4 Jahren zu empfehlen.

Die pharmakologische Zubereitung ist zudem ein Problem: Viele der Phytotherapeutika enthalten Ethanol. Die HMPC hält es für problematisch, Kindern Alkohol zu geben, und gibt als Grenzwert eine Konzentration von 0,125 Promille nach der Verabreichung an; 1 Promille gilt bereits als toxisch. Nach den Empfehlungen der HMPC sollten Kinder unter 2 Jahren keine alkoholhaltigen Arzneimittel bekommen, wenn nicht eine zwingende Begründung vorliegt. Die Behandlungsdauer bei Kindern unter 6 Jahren sollte auf eine Woche beschränkt werden.

Für Probleme bei der Verwendung von Phytotherapeutika in der Kindermedizin ist die EU-Verordnung EC1901/2006 verantwortlich, die die Zulassung von Heilmitteln für Kinder regelt. Das Ziel der Verordnung ist es, dafür zu sorgen, dass Kinder nur mit hochwertigen, sicheren und wirksamen Arzneimitteln behandelt werden. Seit Anfang 2007 ist sie in Kraft. Für die Schweiz hat sie keine direkten Auswirkungen, doch indirekt werden sich die in der Europäischen Union gefällten Entscheidungen über die $\mathrm{Zu}$ lassung von Präparaten von Kindern auch hier bemerkbar machen.
Bei der Zulassung von Präparaten bei Kindern werden besonders hohe Anforderungen an die Sicherheit und die Wirksamkeit gestellt, die weit über die Standards hinausgehen, die bei Erwachsenen gelten. Mit den Zulassungsunterlagen muss ein pädiatrisches Studienkonzept vorgelegt werden, aus dem die geplanten Prüfungen für die fünf in der Verordnung festgelegten Altersgruppen hervorgehen müssen. Die Bestimmung, dass die Studien zur Sicherheit und Wirksamkeit an Kindern verschiedener Altersgruppen durchgeführt werden müssen, erhöht den Aufwand beträchtlich und treibt somit die Entwicklungskosten des Präparats in die Höhe.

Ausnahmen von dieser strikten Regelung sieht die Verordnung nur bei Generika, bei traditionellen pflanzlichen Heilmitteln und bei Wirkstoffen vor, die biologisch bereits anerkannten sehr ähnlich sind. Klar ist, dass neue Erkenntnisse der Phytotherapie bei einer solchen Ausgangslage nur eine geringe Chance haben, in die Kinderheilkunde Eingang zu finden. In der Pädiatrie sind die Krankheitsbilder in vielen Fällen schwer voneinander abzugrenzen. Das gilt insbesondere für die Beschwerden, bei denen pflanzliche Präparate häufig eingesetzt werden. Problematisch ist es auch, eine ausreichend grosse Zahl von Kindern in den verschiedenen Altersstufen zu finden, da die Fallzahlen in der Pädiatrie meist kleiner sowie die juristischen und ethischen Probleme gross sind, Kinder in Studien einzubeziehen: Sie können ihre Einwilligung nicht selbst geben, während die Eltern oft zögern, solchen Versuchen zuzustimmen. (rfi) 